\title{
Technical artifacts: An integrated perspective
}

\author{
Stefano Borgo ${ }^{\mathrm{a}, *}$, Maarten Franssen ${ }^{\mathrm{b}}$, Paweł Garbacz ${ }^{\mathrm{c}}$, Yoshinobu Kitamura ${ }^{\mathrm{d}}$, \\ Riichiro Mizoguchi ${ }^{\mathrm{e}}$ and Pieter E. Vermaas ${ }^{\mathrm{b}}$ \\ ${ }^{a}$ Laboratory for Applied Ontology, ISTC-CNR and KRDB FUB, Trento, Italy \\ ${ }^{\mathrm{b}}$ Philosophy Department, Delft University of Technology, Delft, The Netherlands \\ ${ }^{\mathrm{c}}$ Department of Philosophy, John Paul II Catholic University of Lublin, Lublin, Poland \\ ${ }^{\mathrm{d}}$ Institute of Scientific and Industrial Research, Osaka University, Osaka, Japan \\ ${ }^{\mathrm{e}}$ Research Center for Service Science, School of Knowledge Science, \\ Japan Advanced Institute of Science and Technology, Komatsu, Japan
}

\begin{abstract}
Humans are always interested in distinguishing natural and artificial entities although there is no sharp demarcation between the two categories. Surprisingly, things do not improve when the second type of entities is restricted to the arguably more constrained realm of physical technical artifacts. This paper helps to clarify the relationship between natural entities and technical artifacts by developing a conceptual landscape within which to analyze these notions. The framework is developed by studying three definitions of technical artifact which arise from different perspectives. All these perspectives share two intuitions: that technical artifacts are physical objects that exist by human intervention; and that technical artifacts are entities to be contrasted to natural entities. Yet the perspectives are different in the way they spell out these intuitions: the relevant human intervention may range from intentional selection to intentional production; and the contrast between technical artifacts and natural entities may be introduced by a constitution relation or by defining properties that set technical artifacts apart. The three perspectives are compared and their similarities and dissimilarities are explored with the help of ontological analysis.
\end{abstract}

Keywords: Technical artifact, formal ontology, natural-artificial distinction

Accepted by: Nicola Guarino

\section{Introduction}

Technical artifacts are all around us. We have them at hand's reach, such as chairs, at a distance, such as power plants, and at far distances, such as satellites. By this omnipresence technical artifacts have been incorporated in ontology, ranging from ontologies of specific consumer products to general ontologies for technical components and materials. From a more formal ontological perspective this incorporation leads to also including a general notion of technical artifact. But capturing the notion of technical artifact, and relating it to other notions such as that of natural entity and other notions of artifacts like social, institutional, and artistic artifacts, is not a straightforward task. There are strong intuitions available about technical artifacts which may help capturing this notion. Technical artifacts are seen as physical objects that exist by human intervention, where this intervention involves precise reasons for selection or for production, primarily to adapt the physical environment to our needs and desires. And technical artifacts are typically contrasted to natural entities. Yet, as we shall see, there is

\footnotetext{
* Corresponding author: Stefano Borgo, LOA, ISTC-CNR, Via alla Cascata 56/C, Povo I-38100, Trento, Italy. Tel.: +39 0461 314873; Fax: +39 0461 314875; E-mail: stefano.borgo@cnr.it.
} 
more than one way in which these intuitions can be incorporated into a characterization of what technical artifacts are.

This paper aims at a contribution to the task of incorporating the general notion of technical artifact in applied ontologies, and at addressing the relation between technical artifacts and natural entities; the relation between technical artifacts and other types of artifacts, like works of art, is not addressed. The paper will bring together three perspectives on what technical artifacts are, capture these perspectives with definitions cast in a common terminology, and then compare the perspectives. The three perspectives have their origins in, respectively, applied ontology, engineering design and philosophy of technology, and lead to three definitions that put emphasis on different aspects. A technical artifact is, according to the first, a physical object created by an intentional selection (not necessarily via production) of a material entity as the constituent of the artifact, and the attribution of a technical quality. In the second case, a technical artifact is a physical object that has been intentionally produced and is expected to realize some intended behavior when participating in given technical situations. In the last definition a technical artifact is also the result of an intentional production which, in this case, has to be based on some explicit make plan, the artifact has to correspond to some previously given physical description and, finally, there must be a use plan for the technical artifact.

Throughout the paper we label the categories corresponding to these views with Ontological Artifact, Engineering Artifact and Technological Artifact, respectively. These perspectives need not represent the dominant views within their original domains, yet we submit them as giving three major ways in which technical artifacts can be understood.

The paper, a development and improvement of the analysis in Borgo et al. (2011) including some corrections, is organized as follows: Sections 2-4 introduce the three notions of technical artifact, and sketch the contexts and motivations by which they emerged in applied ontology, engineering design, and philosophy of technology. Section 5 analyses the ontological notions on which the definitions rely and looks at ontological commonalities across the definitions. Section 6 presents an integrated framework that shows several relations among the three definitions and allows us to identify where they agree and disagree. In Section 7 we take some distance from the details of the definitions and continue the comparison in a more exploratory way. Section 8 looks at a fourth definition in the literature and shows how it compares with those presented here. The final section highlights how the results in this paper advance the knowledge on artifact categorization and adds some final observations.

As said, the next three sections collect and motivate the proposed definitions. The goal is to briefly introduce the general perspective of the authors, to rephrase each definition in a common setting that is suitable for the comparison, and finally to describe the intuitions behind each definition.

\section{Technical artifacts in applied ontology}

This section describes the definition of technical artifact proposed by researchers within the Laboratory for Applied Ontology ${ }^{1}$ (LOA) of the Institute of Cognitive Sciences and Technologies in the National Research Council (CNR). Overall, the goal of the LOA group is to apply ontological analysis to study and develop theories of commonsense reality. This general perspective is well represented by the DolCE ontology, see Masolo et al. (2002) and Borgo and Masolo (2009). DoLCE is a foundational ontology used in everyday situations as arising in e-government, manufacturing, information systems

\footnotetext{
${ }^{1}$ http://www.loa.istc.cnr.it/.
} 
and socio-technical systems in general. The approach is human centered and does not aim to be directly applied to specialized areas like, say, nuclear and particle physics. The broad research goal of the LOA group requires to develop ontological understanding of a variety of general notions that are of interest across disciplines. Within this group, particular attention has been paid to the notions of artifact and of role since these turn out to be at the core of most entities we recognize in social reality and sociotechnical systems.

\subsection{Background of the definition}

The notion of artifact here developed has been studied from the perspective of applied ontology, and is therefore given as a normative notion justified by ontological considerations. A formal definition has been given in first order logic by taking the DOLCE ontology framework as the background theory but the motivations, the analysis and the overall approach are largely independent from DOLCE itself. Indeed, the developed definition and surrounding conceptual framework is intended as a general ontology-based theory of artifacts and relies on a set of other ontological notions, like agent, event, and intentionality, which here are not discussed.

\subsection{Definition}

This notion of artifact was introduced and formally developed by Stefano Borgo and Laure Vieu in Borgo and Vieu (2009). One can rephrase the formal definition as follows: An artifact $\mathfrak{a}$ is a physical object which an agent (or group of agents) creates by two, possibly concurrent, intentional acts: the selection of a material entity (as the only constituent of $\mathfrak{a}$ ) and the attribution to $\mathfrak{a}$ of a quality. ${ }^{2}$ The definition is more general than those discussed in application domains in several aspects. In particular, an artifact does not need to be the outcome of a production process as usually understood, i.e., a physically modified entity. According to this view, an artifact may arise when a material entity, say a pebble, is selected to create a new entity, say a paperweight, by attributing to it a quality, namely the ability to perform as a paperweight. The new entity, the artifact, is constituted by the selected material entity but has an additional property, the attributed quality to perform as a paperweight, that the material entity, the pebble, does not have.

The new entity, the artifact, is constituted by the selected material entity and, with respect to it, has a new distinct property, namely the attributed quality to perform as a paperweight. Of course, produced items are classified as artifacts by the definition since the design process as well as the intentional production process are specific ways to select an entity (what is produced) as the ontological constituent of an artifact and to create the particular artifact by the act of attributing a desired quality, namely the quality that characterizes that kind of artifacts: a produced knife is created when a material entity resulting from intentional manipulation is selected as the constituent of an artifact and the cutting-quality is attributed to such an artifact.

The definition refers to material entities as constituents of artifacts. The term material entity is intended to collect both physical objects and amounts of matter since the artifact creator(s) may select a physical object, e.g., a stone, to make paperweight and a specific amount of matter, e.g., some given flour, to make dough (Borgo \& Vieu, 2009). The distinction, in turn, allows to explain why not all artifacts are altered by the same kinds of change. For example, an artifact constituted by a physical object

\footnotetext{
${ }^{2}$ The original definition refers to qualities or capacities suggesting that the classification of properties in the ontology can make a distinction between these two types. For the sake of simplicity, in this paper we only refer to qualities.
} 
may lose parts or have components substituted, provided this fact does not affect the existence of its constituent; this sort of change has different consequences if the artifact is constituted by an amount of matter.

Definition 1 (Ontological artifact). A technical artifact $\mathfrak{a}$ is a physical object which an agent (or group of agents) creates by two, possibly concurrent, intentional acts: the selection of a material entity (as the only constituent of $\mathfrak{a}$ ) and the attribution to $\mathfrak{a}$ of a technical quality.

Note that the definition does not aim to describe the creation event itself. It simply points out the necessary and sufficient conditions for a (technical) artifact to be created and at the same time classifies it from the ontological viewpoint. The collection of technical artifacts identified by this definition includes produced artifacts as well as other artifacts obtained by intentionally collecting natural resources like intentionally dug up rare earth metal (provided we attribute it a technical quality), which is by no means produced. Furthermore, note that by being the result of a selection and quality attribution, the artifact is distinguished from its constituent object or material: a wooden rod and a measuring rod are co-localized yet distinguished entities. Indeed, the first constitutes the second so that the measuring rod does not exist unless the wooden rod exists, but not vice versa. It should also be noted that there is no claim that the artifact has that technical quality or that it performs according to it. A measuring rod might turn out to be too badly marked to function as such. In this case one says that the artifact exists and is malfunctioning. Also, notice that some quality attributions are parts of a shared social perspective which justifies why the corresponding artifacts are publicly recognized as such, e.g., cars, buildings and knives. Finally, the definition implies that the actual use of the artifact is irrelevant for its ontological status.

\subsection{Discussion}

The definition leaves aside the issue of describing what distinguishes a technical artifact from other senses of artifact as used in, for example, biology, social sciences, or aesthetics. This description is delegated to a specification of the meaning of technical quality not undertaken here since it serves merely to restrict the definition to technical artifact rather than artifact generally. The goal is to isolate crucial elements, like intentionality, that must be present in order to properly understand artifacts. The basic idea is that an artifact is something existing because an agent intentionally brought it into being. In the most common examples, like cars and knives, this intentionality is part of a production process. However, the discussion of what effects a production must have on the material at stake in order to turn it into an artifact shows that what counts is the bare presence of a physical manipulation, not the effects of it. This observation leads to consider the role of the production of an artifact as a demarcation point that sanctions the creation of the artifact itself. Ontologically, it is then natural to look at the physical production as a placeholder for the ontologically relevant event of creation. Since in some cases no sensible effect can be attributed to the production itself, it may make sense to claim that the creation of an artifact is not necessarily the result of a physical act: it is instead an intentional act which in many cases is performed via a physical production. Once the import of intentionality in the notion of artifact has been clarified, agentivity and creation find their place in the definition to ground the presence and the effect of the intentional act in the generation of the new entity. In order to distinguish the material entity from the artifact, the authors make use of the ontological relation of constitution as understood in DoLCE (Masolo et al., 2002) as well as of the "entity stacking" technique (Vieu et al., 2008).

Being intentionally created is however not enough to be a technical artifact. One can intentionally break a tree branch or intentionally leave a mark on a wall while strolling around. Neither the branch nor 
the mark are per se artifacts, at most one would classify them as artifactual entities. The intuition here is that the artifact created from the intentionally selected entity must bear something new with respect to the original material entity; the branch is broken so that it is (or seems to be) long enough to reach a fruit, the mark is made so to be visible to fellows. The creation of the artifact is thus motivated by the agents' intention to create something that has some given quality. This analysis shows that the creation of an artifact is more than an intentional selection of a material entity, it is the selection of an entity to generate something which is taken to satisfy or possess a specific quality. Once these crucial elements are linked together, a notion has been reached that the authors propose as identifying the ontological category of artifacts. From here, one can start discussing how much this notion can be specialized to make sense of what happens in specific domains or for specific applications, in particular what makes an artifact a technical artifact rather than some other kind of artifact.

\section{Technical artifacts in engineering design}

This section describes the definition of technical artifact proposed by Riichiro Mizoguchi and Yoshinobu Kitamura while both at the Mizoguchi Lab in the Institute of Scientific and Industrial Research at Osaka University. The Mizoguchi Lab has been working since the 1990s on functionality of artifacts from an engineering point of view (Kitamura \& Mizoguchi, 2010). Function as foreseen by the designer is taken as a crucial aspect of engineering artifacts. By looking at a (natural) stone and a factory-made paperweight, one reaches an intuitive distinction between artifact functions and user functions, and this intuition leads to the definition of technical artifacts presented in this paper.

\subsection{Background of the definition}

The notion of technical artifact developed at the Mizoguchi Lab relies on engineering design research. The main idea is based on clear distinctions between the design, manufacturing, and use phases of the life cycle of artifacts. These distinctions are typically observed for engineering artifacts, but are believed to be applicable to other kinds of artifacts as well, even if some phases could be implicit for such artifacts. In addition, the design and the production phases play a crucial role to identify technical artifacts. Although the study of artifacts in engineering design has been the first motivation for the work at the Mizoguchi Lab, later the target has been extended and now the notion covers essential conditions for a wider class of technical artifacts.

\subsection{Definition}

The notion of technical artifact proposed by Kitamura and Mizoguchi is as the following definition.

Definition 2 (Engineering artifact). A technical artifact $\mathfrak{a}$ is a physical object created by an intentionally performed production process. The process is intentionally performed by one or more agents with the goal of producing the object $\mathfrak{a}$ which is expected to realize intended behavior in some given generic technical situation, and the object $\mathfrak{a}$ can realize to some extent that intended behavior and/or has a property which supports that behavior.

The expression 'intentionally performed production process' means that the agent(s) intentionally performs the process which results in physical changes (these changes can be at the chemical or topological 
level as well, including assembling) from which the object $\mathfrak{a}$ is produced. The goal of the agent who executes the process is that the produced object $\mathfrak{a}$ will realize (exhibit) a specific behavior under a given situation, where by behavior it is meant a relationship between the artifact and the environment, typically an input-output relationship on some measurable parameters. Under such a situation, the behavior of the artifact $\mathfrak{a}$ manifests $\mathfrak{a}$ 's essential function ${ }^{3}$ (Kitamura \& Mizoguchi, 2010) since it is intended by the agent as the creator of that object. The situation of a technical artifact in which its function is performed is typically given by use of the artifact. It can be categorized into two cases: (1) an artifact such as a cup is used as a whole by a user externally, and (2) an artifact such as a bolt is used as a component in a larger artifact. In the Kitamura-Mizoguchi theory, see Kitamura and Mizoguchi (2010), a function is performed in either of these situations (called 'function contexts' in that paper, for users directly or for the whole system's function, respectively). Note that the expression 'generic technical situation' in the definition means that it is not a specific situation of use but a generic users' situation which is not fully specified by the agent.

According to this definition, object $\mathfrak{a}$ is a technical artifact even if it can only partially realize the behavior intended by the agent at the beginning of the production process, under the condition that there are identifiable clues of the initial selected quality. For example, consider the case of an agent that does not complete the production process, or the case in which the produced object is (partially) defective for some reason, or even the case of a produced object that realizes only behaviors which to some extent differ from what was initially intended by the agent: in all these cases the agent still has produced a technical artifact according to this definition as long as the resulting object is in some (relevant) way sufficiently close to the intended outcome. Finally, a user may use the artifact $\mathfrak{a}$ differently from how the creator intended it without altering the artifact status of $\mathfrak{a}$.

\subsection{Discussion}

From an engineering point of view, the manufacturing phase (and the design phase) play an important role in identifying technical artifacts. The manufacturing act is regarded as an act to embody "intrinsic" physical qualities (e.g., shape, material and topology) which are not easily changed. The definition of engineering artifact fits this naive understanding. It depends only on the production process in the manufacturing phase based on the intention in the design phase. Such production processes are also based on a structured and reasoned approach in the design phase for embodying such physical qualities that realize the intended behavior. The behavior is a "realization" rather than the capacity based on those physical qualities.

Artifacts are independent of any other entities (as human beings are) except their parts as explained below. From the starting timepoint of physical existence of a technical artifact in this view, the object has intrinsic physical qualities, in which roles intended by the agent inhere to realize the intended behavior for the intended situation. Actions in the actual use phase have no effect on the identity of the object $\mathfrak{a}$. In other words, whether or not something is an artifact, and if so what kind it is, is independent of the actual use situation.

\footnotetext{
${ }^{3}$ The definitions of the notion of function and the distinction from behavior have been extensively discussed in the literature, see Burek (2007) and Burek et al. (2009). In the theory of function developed by Kitamura and Mizoguchi (2010), function is distinguished from behavior since the first is context-dependent. A behavior is a kind of a physical process performed by an artifact as a device. A function is defined as "a role played by a (device-oriented) behavior in a teleological (function) context". Since this issue is not central to this paper, we refer the reader to Kitamura and Mizoguchi (2010) for the detailed definition and discussion.
} 
The possibility of use in generic situations is also important in engineering. It is realized by a design for generic use and by manufacturing acts for its stable embodiment. Thus, in the Kitamura-Mizoguchi theory, selection of an object for a specific situation is regarded as an act in the use phase. For example, a selection of a branch of a tree (or a table) for one's use as a chair, is regarded as an act in the use phase and then the selected object performs an accidental function which is the same as the essential function of a chair (Kitamura \& Mizoguchi, 2010).

\section{Technical artifacts in philosophy of technology}

This section describes the definition of technical artifact proposed by researchers of the Philosophy Department of Delft University of Technology within the research program called The Dual Nature of Technical Artifacts carried out between 2000 and 2005 (Kroes \& Meijers, 2006). This program aimed at addressing a number of philosophical issues about artifacts, and for doing so the concept of technical function and the activities of the design and use of physical objects were analyzed in terms of use plans, see Houkes and Vermaas (2010). In related work on the metaphysics of artifacts (Houkes \& Vermaas, 2009a, 2014), the notion of make plan was introduced for describing manufacturing and production. These descriptions of design, use, manufacturing, and production by means of use plans and make plans are to some extent rational reconstructions, adding detail to the descriptions that may remain implicit in more common descriptions of design, use, etc.

\subsection{Background of the definition}

Wybo Houkes and Pieter Vermaas in (Houkes \& Vermaas, 2014) define artifacts as physical objects that are produced by carrying out make plans, and this definition is the result of a conceptual analysis of engineering manufacturing and production practices, and of an evaluation of the different options for defining artifacts within the plan analysis (Houkes \& Vermaas, 2009a). This plan analysis originated in a characterization of designing and using in terms of use plans (Houkes \& Vermaas, 2010).

A use plan for a physical object is a goal-directed series of considered actions, where some of the actions involve interacting with the object. ${ }^{4}$ The use of a physical object by an agent can then be described as the carrying out of a use plan for that object, i.e., as the carrying out of the considered actions of the plan by the agent, including the actions that let the agent interact with the physical object, aimed at realizing the goal associated to the use plan. Plan design can be described as the development of a use plan for a physical object (or objects) for realizing the specified goal. And product design can be described as giving the physical description of the objects involved in the use plan. Finally, a technical function of a physical object can be characterized relative to the object's use plan, as the capacity for which (i) the object is designed in product design, and that (ii) is justifiably assumed to contribute to the realization of the goal of the use plan when that plan is carried out (Houkes \& Vermaas, 2010).

The option to define general artifacts as physical objects for which use plans exist was not adopted by Houkes and Vermaas for two general reasons. First, this option would imply that natural entities for which uses are defined, such as pebbles and tree trunks, would become artifacts as well. This implication was taken as violating the everyday distinction between natural and artificial entities, and the assumption that engineering produces such artificial entities. Second, defining technical artifacts as physical

\footnotetext{
${ }^{4}$ Interactions with objects can be physical manipulations of these objects, but may also be remote causal interactions (e.g., accelerating space probes by means of Jupiter's gravitational field) and observations (e.g., watching traffic lights).
} 
objects with use plans would introduce an unwanted dynamical aspect to the categorization of artifacts: whenever an agent develops a new use plan for an existing object, say for a car, when parents decide to lull their infants to sleep by driving them around, that object would instantaneously enroll in the corresponding new category of "soothing" artifacts (see also Carrara \& Vermaas (2009)).

A make plan is in turn a use plan for a set of materials and a set of tools that has the aim to create a physical object $\mathfrak{a}$ that meets a specific physical description id. Carrying out a make plan for a physical object $\mathfrak{a}$ counts as producing $\mathfrak{a}$, and a make plan is developed by manufacturing engineers on the basis of the physical description id as determined in product design of $\mathfrak{a}$ (Houkes \& Vermaas, 2009a, 2014). In engineering design this physical description is typically given in an explicit manner, yet may in more common-and-garden production be left implicit. In the plan analysis the physical descriptions id are assumed to be always explicit, which shows that in some cases the analysis presents a rational reconstruction of production.

\subsection{Definition}

The general notion of artifact proposed by Houkes and Vermaas (2014) is as follows: An artifact $\mathfrak{a}$ is a physical object created by the carrying out by an agent (or group of agents) of a make plan for a physical object with a physical description id. Given the topic of this paper this characterization is still too wide, as was the ontological definition proposed for general artifacts and specialized in Section 2. A focus on technical artifacts is achieved within the plan analysis by defining them as artifacts for which there exist one or more use plans, thus singling out technical artifacts as made physical objects that can be employed to realize goals.

The resulting notion of technical artifact then is the following definition.

Definition 3 (Technological artifact). A technical artifact $\mathfrak{a}$ is a physical object which is, firstly, created by the carrying out by an agent (or group of agents) of a make plan for a physical object with a physical description $i d$, and for which, secondly, a use plan exists.

A requirement on make plans is that one or more of the materials is physically modified by a change in their physical properties, or that two or more of these materials are assembled, or both. This requirement rules out that a plan to merely intentionally select an entity that meets a physical description $i d$ can be taken as a make plan for such an entity; selection does not count as creating a technical artifact on this view. This requirement also makes that the set of natural entities and the set of technical artifacts are disjoint: an entity that comes into existence by a natural process may be a natural entity but is not a technical artifact; an entity that comes into existence by an agent carrying out a make plan, may be a technical artifact but is not a natural entity. It is thus a historical fact whether an entity is a technical artifact or not, and for a technical artifact it is a historical fact for what physical description id it was produced. By now categorizing technical artifacts by means of their $i d$, it follows that a dynamics in their categorization is absent: when a new use plan is developed these technical artifacts do not change their membership to a specific category $i d$.

Standards of (practical) rationality on plans (Houkes \& Vermaas, 2010, Section 2.6) lead to the further requirement that there is a reasonable chance that a make plan for an object with a physical description $i d$, when carried out, indeed yields an object of that description. One may accept that make plans yield probabilistically the intended objects. The first procedures for producing flat television screens often led to screens with little flaws. Yet a make plan that never succeeds in creating objects with the intended 
physical description $i d$ can rationally speaking not be taken as a make plan for such objects. Moreover, when in manufacturing objects are created that have only probabilistically the required physical characteristics, actions are included in which the created objects are checked against the physical description for which they were produced. This checking action is assumed to be part of a make plan, which means that physical objects created to have a physical description id yet fail to do so, are not technical artifacts.

\subsection{Discussion}

By defining the general category of artifacts by means of make plans the connection between use and artifactuality is loosened on this view (Houkes \& Vermaas, 2009b). Artifacts may be typically physical objects with use plans, yet need not be so. Chairs, Diesel engines, and nuclear submarines are artifacts and also objects with use plans, and thus also technical artifacts. Transuranic atoms and the largest sandcastle on the beach, are artifacts but not always objects with use plans. Conversely, pebbles used as paperweights and fallen tree trunks used for crossing streams are physical objects with use plans but not artifacts and thus not technical artifacts.

As said, the set of natural entities and the set of technical artifacts are disjoint. But they are not exclusive; according to this definition there are entities that are neither natural entities nor technical artifacts. Artifacts without use plans are such entities. Waste products are cases, being entities that are the result of intentional action but not of carrying out a make plan for those entities. And so are the entities that are intentionally created by carrying out make plans yet failing to meet the physical descriptions id specified in the plans.

Whether or not a physical entity is a technical artifact of some type $i d$ is an ontological and historical fact. The physical structure of an object may from an epistemic point of view be a criterion for establishing this fact. Many physical descriptions id that characterize types of technical artifacts entail that natural entities cannot reasonably meet such descriptions, allowing the conclusion that an entity that does meet such a description $i d$ is indeed a technical artifact. Yet this does not hold in general. The physical structure of an artificial diamond, a technical artifact according to Definition 3, may be exactly similar to the physical description of a natural one, meaning that it cannot be deduced from the physical description of such a diamond that it is a technical artifact.

\section{The ontological core of technical artifacts}

The three approaches we have described in the previous sections put emphasis on a variety of aspects and are leading to notions of technical artifacts that seem to have little in common except, perhaps, that they all capture our shared intuition toward undisputed examples: from chairs to violins, from telescopes to brooms. Indeed, Definition 1 allows artifacts to be created without production, Definition 2 takes it as an essential ingredient, while Definition 3 requires the existence of a physical description which is ignored in both other approaches.

When notions are so different, one usually concludes that they should be treated as leading to separate concepts and then discusses whether they could be considered as extensions of a core notion and if the latter may actually exist. Even worse, if we further expand our considerations beyond these three notions and include other approaches in the large literature on artifacts, ${ }^{5}$ we would easily conclude that

\footnotetext{
${ }^{5}$ Another possibility is that the notion of artifact is not a concept in the sense of the so-called classical theory of concepts, but rather a family resemblance concept in the Wittgensteinian sense. Carrara et al. (2011) explore this possibility for the case of technical functions, which are closely related to artifacts.
} 
our commonsense intuitions on artifacts do not lead to solid grounds for a formal characterization of a concept of technical artifact.

In this paper, however, we show that this conclusion may change when the three approaches are analyzed with ontological techniques. When the Definitions 1, 2 and 3 and the explanations of how they are to be applied are interpreted within a single foundational ontological framework, it can be argued that they rely on a set of shared notions. For giving this argument we adopt DoLCE as our single foundational ontological framework and then characterize the three approaches in terms of notions provided in DOLCE. These characterizations can be taken as specifying the three definitions of technical artifacts ontologically, yet they are for Definitions 2 and 3 in principle interpretations: Definition 1 is already formulated in the DOLCE framework, but the two other definitions are formulated within their own original conceptual frameworks different to DOLCE, and may be precise or less precise in their wording relative to DOLCE standards; the characterizations of Definitions 2 and 3 are therefore proposals for capturing them within the DOLCE framework. But notwithstanding this caveat, this exercise shows that the three approaches to technical artifacts can be taken as relying on a number of core elements informally identified as DOLCE notions. Six DOLCE notions occur in the characterizations of all three definitions, namely:

- Physical entity;

- Action;

- Physical property; ${ }^{6}$

- Agent;

- Selection (mental);

- Intentional physical production.

Moreover, the characterizations of Definitions 1 and 3 share the notions Attributed physical property. Figure 1 lists the ontological categories used in the three characterizations and shows which characterization relies on which category, including ones that are specific to characterizations.

We proceed as follows. First we identify these core elements and provide a general description of their meaning, and use them as building blocks for the formal characterizations of the three approaches to technical artifacts. Indeed, we aim to show that the three approaches can be understood as specific combinations of the above notions. As a result of this, the next section presents an ontological comparison of the approaches underlying Definitions 1-3 based on how they integrate different notions to capture a specific understanding of the term 'technical artifact'.

This strategy, of course, is not limited to the three approaches we analyze here and we will give an example of this by using the same framework to discuss a fourth approach towards artifacts in Section 8 . We indeed surmise that this strategy can be broadly used to compare different characterizations of the notion of artifact.

As anticipated, there is only a generic agreement on the characterizing aspects of the basic categories and we make the simplifying assumption that, beside the DOLCE framework, the undefined categories in Fig. 1 are dealt with in generic terms. For example, we assume only broadly accepted viewpoints about agents, namely that these are entities with intentionality and that can intentionally act in the environment to pursue their goals. These are indeed the characteristics given in DOLCE and we avoid any more specific claim about the nature and properties of agents. More generally, we assume that the precise

\footnotetext{
${ }^{6}$ In DoLCE properties are individual qualities. Since this specific choice in understanding properties does not affect the analysis, for the sake of simplicity here we use the generic term 'property'.
} 


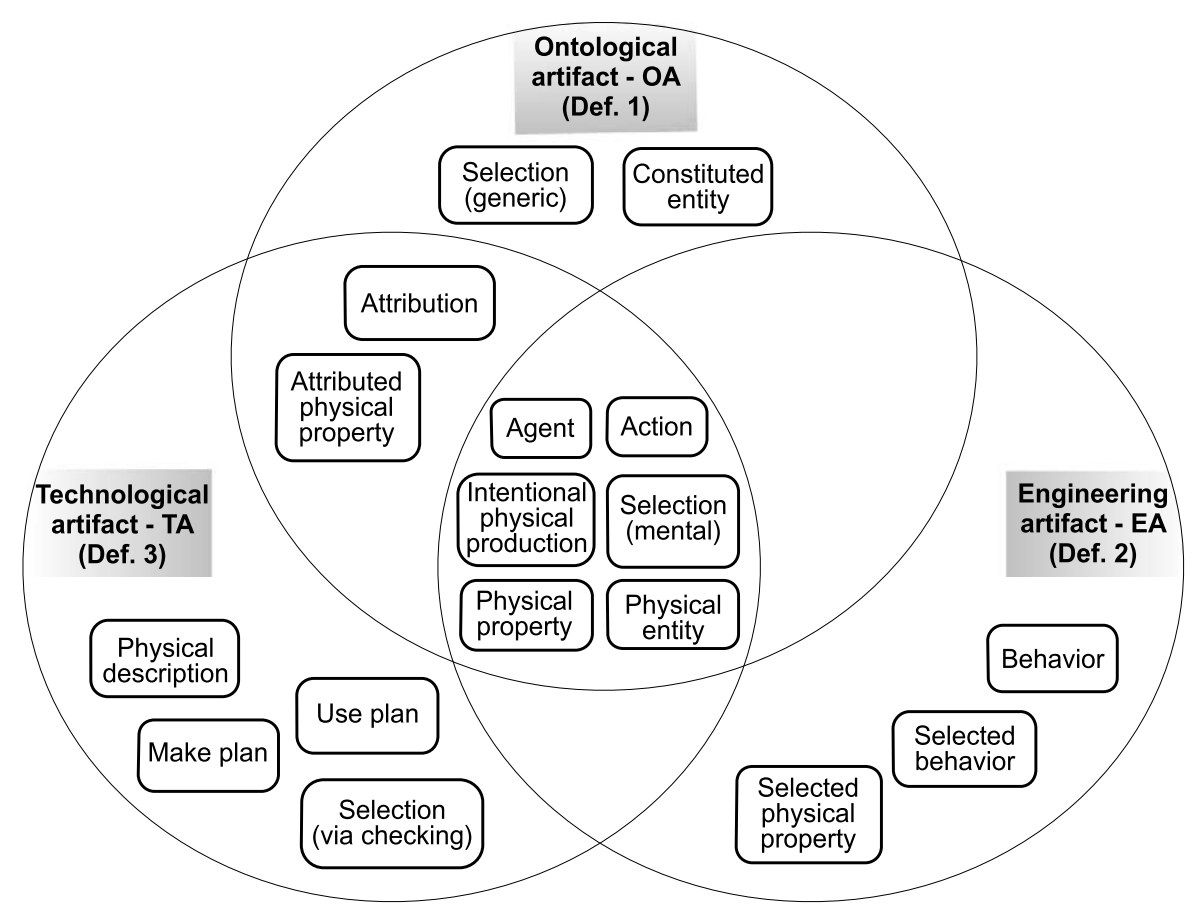

Fig. 1. Venn diagram showing the main categories on which Definitions 1, 2 and 3 rely. The categories are broadly understood. Note that the category Selection (general) is a supercategory of Selection (mental) and Selection (via checking) (the latter do not need to be disjoint).

extension of the Agent category is not fixed in advance but depends on the ontological framework within which one decides to instantiate the analysis. We can thus say that for us these categories are placeholders for the actual ontological categories supplied by an ontological framework, i.e., DOLCE in this paper. At this point we should clarify that Definition 1, although explicitly formalized in the framework of the DOLCE ontology, has been explicitly developed by the authors to be used independently of this ontology (Borgo \& Vieu, 2009).

As mentioned in the Introduction, there is a common understanding that artifacts exist because of some form of intentional (mostly human) activity. In all the definitions intentionality can be detected in physical and mental actions. Definition 1 relies on actions like selection and attribution which can be mental and/or physical. Definition 3 also has mental and physical actions: this definition refers to mental actions like checking ${ }^{7}$ and to physical actions like producing. Note also that the notions of make plan, of use plan and of physical description themselves are intrinsically dependent on intentionality. Actions in Definition 2 are just physical actions seen as components of the physical production process; yet the definition refers to selected behavior as well as to a selection of relevant situations (dubbed 'generic' and 'technical'), all elements that rely on mental actions. Since the reference to the categories of agent, physical entity, and property are clear, these observations suffice to justify the six ontological categories shown as common to all definitions in Fig. 1.

\footnotetext{
${ }^{7}$ A checking action, as understood commonly, can be a mix of physical and mental actions. Here we distinguish these two aspects and assume that a checking action is necessarily a mental action consisting in deciding that the observed property is within the acceptable range. The conditions for this action to be performed (the associated physical action) are a different issue as when a person responsible for the checking entrusts a machine to establish the result of the check.
} 


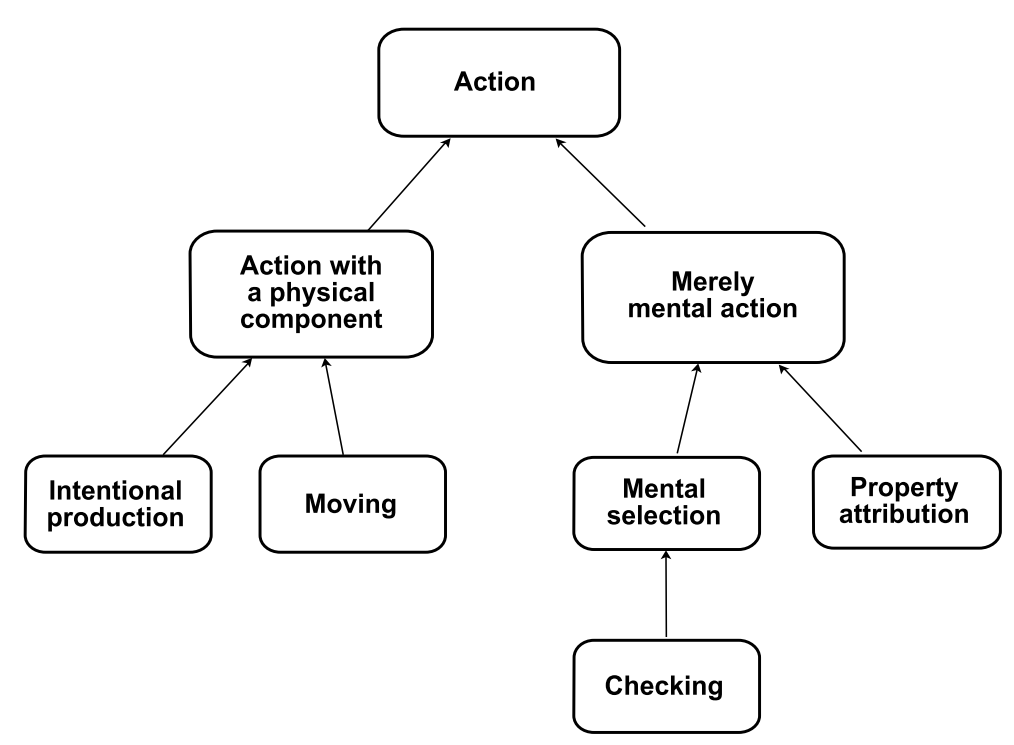

Fig. 2. The action taxonomy relating categories in Fig. 1 according to the study of Definitions 1-3.

One can further analyze a notion used in the definitions to understand finer distinctions and commonalities. In general, this is achieved by subdividing the focussed category according to relevant properties. Regarding actions, we started from the basic distinction between physical vs mental actions as this arises even from the basic examples considered by each perspective. We can go on with the analysis and build another step resulting in the taxonomy of Fig. 2 (left) where we see that Physical action has Intentional production and Moving as subcategories and Mental action has Checking, Attribution and Selection. As argued for checking actions, also property attribution and selection are primarily mental actions. We also observed that all the definitions include, perhaps implicitly, the mental action of selecting the physical entity from which the artifact is created. We will see in the next section that further refinements are needed to properly compare the definitions. For instance, intentional production in Definition 2 can be seen as a particular way to implement the intentional selection mentioned in Definition 1.

Definition 3 implicitly refers to the notions of physical property and attributed physical property due to the checking action aiming to establish that the artifact satisfies a given physical description. We can now identify physical property in the sense of Definition 3 as a subcategory of property to which Definition 1 refers.

The comparison as depicted in Fig. 1 shows an interesting relationship between Definition 2 and Definition 3. In the latter, an entity is taken as an artifact by checking whether a series of actions (ideally) based on a make plan has led to an entity that fits a given physical description. This physical description is limited to the physical property level. Definition 2 instead takes the behavioral level to be the relevant one. Thus, while Definition 3 distinguishes between properties and selected properties, Definition 2 draws an analogous distinction across behaviors and selected behaviors.

Note that for each definition Fig. 1 highlights the property (or properties) considered proper of the created artifact, and distinguishes the latter from the physical entity or material from which it has been created. In Definition 2 the new property is expressed in terms of selected behaviors, and Definition 3 implicitly refers to attributed properties via the physical description. The attribution in this latter case is realized via the checking action, it is only after successfully passing the check that the technical artifact is created separating it from any physical entity that simply underwent an intentional process. 
Finally, the three definitions agree on the notion of physical entity although there is an important ontological difference. Definition 1 assumes that the physical entity - object or material - persists even after the artifact has been created, the physical entity is said to constitute (to form an ontological substratum for) the artifact. This is not so in Definition 2 where the physical entity is transformed into the artifact at the end of the production process provided this process does not go badly wrong. Similarly, in Definition 3 the physical entity is transformed into the artifact at the end of the production process provided this process satisfies the specifications of the make plan underlying it, including the successful execution of the checking action.

The three characterizations of the Definitions 1-3 in DOLCE are (note that the characterization of Definition 1 is just this definition since it was already developed in the DOLCE framework).

Formal characterization 1 (Ontological artifact). A technical artifact $\mathfrak{a}$ is a physical object which an agent (or group of agents) creates by two, possibly concurrent, intentional acts: the selection of a material entity (as the only constituent of $\mathfrak{a}$ ) and the attribution to $\mathfrak{a}$ of a technical quality.

Formal characterization 2 (Engineering artifact). A technical artifact $\mathfrak{a}$ is a physical object which an agent (or group of agents) creates by an intentional act: the carrying out of a production process with the goal of obtaining a physical object $\mathfrak{a}$ that is expected to realize given behavior or a given property when participating in given generic technical situations.

Formal characterization 3 (Technological artifact). A technical artifact $\mathfrak{a}$ is a physical object which is, firstly, created by the carrying out by an agent (or group of agents) of a make plan for a physical object with a physical description id (which includes performing a checking procedure that establishes that the object sufficiently answers to the description $i d$ ) and for which, secondly, a use plan exists.

The analysis we presented in this section focuses on category matching only. The definitions we are studying differ also at the relational level since these categories are related to each other into an integrated framework. The analysis of these relationships is complicated due to the use of relations not yet well formalized. Relations like "realization" or "carry out" require further study to align and consistently compare them.

\section{An integrative perspective on the definitions}

In this section we provide a general perspective for the comparison of the three definitions - Ontological Artifact (OA), Engineering Artifact (EA) and Technological Artifact (TA). This perspective is summarized in Fig. 3. We remind the reader that, as seen in the introduction of the definitions, several categories are used in the comparison without being formally introduced. For instance, we take the category Physical Entity to comprise only material entities: it thus includes amounts of matter like some milk, sand or iron; as well as physical objects like rivers, wooden rods and cars.

The relationship between OA (Definition 1), EA (Definition 2) and TA (Definition 3) is more articulated. First, let us point out that the current more detailed analysis led, contrary to what claimed in Borgo et al. (2011), to conclude that EA is not a subcategory of TA nor vice versa. Clearly, these categories cover common cases as exemplified by the fact that, according to both, cars and satellites are technical artifacts. TA is not a subcategory of EA since an EA technical artifact has some selected behavior and the 


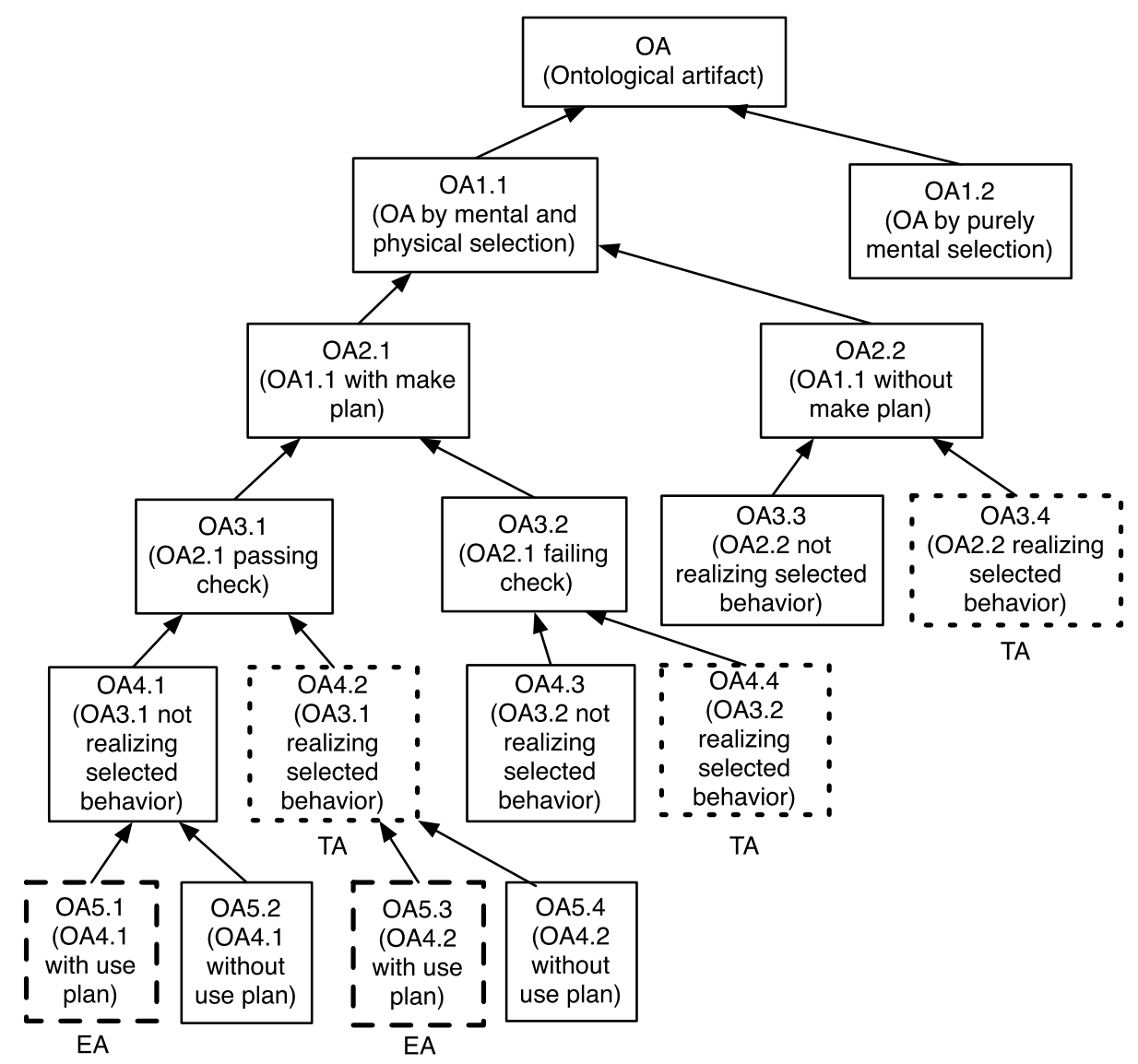

Fig. 3. The (extensional) class of OA artifacts and its division in subclasses following the properties discussed in our analysis (not all levels show the full partition of the superclass). EA and TA extensionally correspond to the union of the subclasses marked by dashed boxes and by dotted boxes, respectively.

definition of TA forces no comparable constraint. As seen in the previous section, we can rephrase this by saying that EA artifacts must have some selected property (e.g., to manifest some relevant behavior).

Also, there are artifacts in the sense of Definition 2 that do not fall within Definition 3. Consider the case of a production process that follows a make plan but that fails to complete and assume that the produced object has the selected behavior at least to some extent. Then, according to Definition 2, this entity is a technical artifact and yet, since the make plan was not entirely carried out, this is not a TA artifact.

As described in Section 2, an element of OA is a physical object constituted by a material entity, that is, another physical object or matter. An OA artifact can be created by selecting any entity (natural, artifactual, or even an artifact) and attributing a property to it. This point is, however, subtle. Both TA and EA are physical objects enriched with a new property related to the intention of the agent who produced the artifact. The selection and property attribution through which an $\mathrm{OA}$ is created runs parallel to the acquisition of the intentional property by TA and EA but follows the stacking methodology typical of multiplicative ontologies (Vieu et al., 2008). What EA and TA view as the acquisition of an intentional property by a physical object is viewed by OA as the creation of a new physical object that is constituted by the given physical object and differs from it mainly for having the intentionally attributed property. 
The relation between OA and EA cannot be one of subsumption. From the ontological perspective underlying $\mathrm{OA}$, the creation of an artifact is accompanied by ontological duplication: a physical object continues to exist, possibly with some of its physical properties altered, or is brought into being by assembling other objects or through the modification of material, and additionally another physical object, the artifact, which has the intentional property of having some property attributed to it, is brought into being. Obviously the former of these physical objects is not an EA, because it lacks the corresponding intentional property. Neither, however, is the latter of these objects an EA, because its history is essentially different: an OA may come into being instantaneously through the mental act of selection and attribution, whereas an EA often comes into being gradually, in the course of a production process.

Nevertheless, although it is not so that whenever there is an OA, there is an EA, but it is true that when there is an EA, there is also an OA. The falsity of the statement that whenever there is an OA, there is an EA follows from the fact that OA does not require any production of a physical object to constitute the artifact-to-be. An OA can come into being by a purely mental act, when an existing object is selected and made into an artifact by having some property attributed to it. This existing object can be a natural object or an object previously created for some unrelated purpose, as when some pebble found on the beach or a broken alarm clock is selected to serve as a paperweight. According to EA, the pebble-asa-paperweight is not a technical artifact, because it did not result from a production process, and the alarm-clock-as-a-paperweight is not an artifact as a paperweight, since it was not produced to exhibit the behavior that is required for serving as a paperweight. From the point of view of EA, an existing EA can be changed into an EA of another kind only by intentionally modifying it to receive new properties that allow it to exhibit behavior fit for its new function. A very simple example is a match that is cut lengthways with a knife to create a toothpick.

The statement that whenever there is an EA, there is an OA needs some clarification. Intentionally carrying out a production process does not necessitate that some quality is attributed to the produced item and nothing forces the production process leading to an EA to be entirely successful. In successful production, the resulting object exhibits the behavior it was intended to exhibit. Then, from the OA perspective, the producing agent selects (or delegates the selection to the production itself) the entity and attributes the intended property to it. In the case of unsuccessful production, the produced object is not capable of exhibiting the intended behavior. The question we have to posit now is whether the object produced by an unsuccessful production is always an EA. Of course, it results from a production process that was aimed at obtaining an object with the capacity to exhibit some behavior. Furthermore, Definition 2 requires that the produced object has to some degree the intended behavior. Indeed, from the definition the object "can realize to some extent that intended behavior and/or property which supports that behavior". Intentional but unsuccessful productions may thus fail to lead to an EA unless the sought property is satisfied at least in part. This condition suffices to conclude that whenever the artifact is created according to EA, a relevant property is identified and attributed (and even present in some measure), so that a corresponding OA exists as well.

In summary, we have it that to each successfully (or partially successfully) produced EA corresponds an OA and to each OA selected by production corresponds an EA. This correspondence is one way we can relate OA and EA as indicated in Fig. 3 by the match between EA and subcategories of OA.

The relation existing between OA and TA is similarly asymmetrical: since TA requires the execution of a make plan, it is not so that to every OA there corresponds a TA. Since every make plan and physical description checking count as a selection and attribution, to every TA corresponds an OA. Finally, this theoretically motivated alignment between TA and a subcategory of OA together with the previous analysis of the alignment between EA and other subcategories of OA, lead us to confirm from the intensional 
viewpoint the overlap between the two categories of EA and of TA, an overlap that we established at the beginning of this section by extensional arguments, i.e., by referring to actual examples.

\section{Similarities and dissimilarities}

The three notions of technical artifact discussed in this paper share the intuition that technical artifacts are to be contrasted with natural entities, but do so in a different way. According to Definition 1 (OA), technical artifacts form a category disjoint from the category of natural entities by a relation of constitution: the intentional act of selection of a natural entity and the property attribution act lead from a natural entity to a new entity, the technical artifact. Following Definitions 2 (EA) and 3 (TA), technical artifacts are not natural entities simple for the reason that they have properties natural entities do not have. In this sense, since technical artifacts are physical objects according to all definitions OA, EA and TA, the notion of physical object embraced in OA differs from that adopted in the other cases.

For EA the distinguishing property of artifacts is the one of being intentionally produced with a goal as characterized in Definition 2, and for TA it is the property of having come into existence by the carrying out of a make plan as specified in Definition 3. The three notions of technical artifact also share the intuition that technical artifacts exist by human intervention, and again spell that out differently. For OA human intervention can be performed as a pure mental act. For EA human intervention is given by the intentional act of production, and for TA it corresponds to the carrying out of a make plan.

Analyzing the definitions of OA, EA and TA in this way gives access to a wider spectrum of possibilities to introduce a general notion of technical artifact in ontology. The OA category is introduced via the constitution relation, and this could alternatively be done by considering the category of physical objects that have the intentional property of being selected for a desired property. Similarly the notions of EA and TA have counterpart notions obtained by adopting the constitution relation.

Hence, at least six general notions of technical artifacts can now be discerned:

(1) introducing technical artifacts via a constitution relation or

(2) via an intentional property;

and, independently of this first choice,

(1) via the intentional acts of selection and property attribution, or

(2) via the intentional production aimed to realize the intended behavior in some generic situation, or

(3) via the intentional carrying out of a make plan.

The first choice of introducing technical artifacts via a constitution relation or via an intentional property represents a fundamental choice in ontology structuring. The second choice concerning the specific intentions that set apart technical artifacts is more subtle. In philosophy of technology and philosophical metaphysics intentions related to selection of entities (as when aimed for use) and intentions related to the making of artifacts are distinguished. Dipert (1993), for instance, discerns three types of artificial entities by means of the intentions of the agents involved: instruments form the broadest type and are entities that have been "intentionally used in intentional activity"; tools form a subtype of instruments: they are instruments intentionally modified for contemplated use; and artifacts proper form a subtype of tools, being modified tools "whose modified properties were intended by the agent to be recognized by an agent at a later time as having been intentionally altered for that, or some other use" (Dipert, 1993, pp. 24-30). Hilpinen, in contrast is less discriminating. He chooses the term 'artifact' to refer to all entities that are intentionally made, and spells out making as physical modification, but then 
also allows entities that are merely expropriated for use without physical modification - his example is driftwood picked up from the beach and put on exhibition as art - as a limiting case of an artifact (Hilpinen, 1992, 1993). If in ontology a similar leniency is allowed concerning the second choice about introducing the general notion of technical artifact, it can be argued that some of the differences between $\mathrm{OA}, \mathrm{EA}$ and TA as introduced in this paper can be made to disappear. The difference between embracing a constitution view or not, remains present; but the difference between the characterizing intentions can be challenged.

The lesson to take home, if we wish to suggest one from the analysis carried out in this paper, is that informal notions like that of technical artifact can be spelled out from a variety of perspectives that lead to distinct definitions. Yet, the ontological assumptions behind them show that these intuitions are only apparently far apart. We have seen that the three definitions here studied take three clear stands: Definition 1 focuses on the agent level (both selection and attribution can be just mental acts); Definition 2 focuses on production to constrain the behavioral level; Definition 3 focuses on production to constrain the property level. The comparison in Fig. 3 tells us that we can cast all these intuitions within a single spectrum where there is one key element, the historical creation/production event, and a few sharp demarcation points: intentional production, production description (make plan), product description (checking) and product behavior. Of course, one can add further distinctions to model particular viewpoints but, ontologically speaking, the core elements on which our intuitions of technical artifacts rely should be now clear.

\section{Technical artifacts in formal ontology: An analysis of Kassel's proposal}

Recently, Gilles Kassel (2010) has proposed formal definitions of artifact and technical artifact aimed at extending ontological systems to cover the artifactual world. In particular, these definitions are given within the framework of the DOLCE ontology just as Definition 1. Note that Kassel's original definition is wider in scope than Definitions 1-3 since it takes into account also non-physical artifacts, i.e., artifacts like a computer program, a literary work or a law. ${ }^{8}$ We can restrict Kassel's definition to the class of physical artifacts as follows.

Definition (from Kassel, adapted). An artifact $\mathfrak{a}$ is a physical entity intentionally and successfully produced. Technical artifacts are artifacts which possess a function, either proper or accidental (Kassel, 2010, p. 224).

First, note the reference to intentionality and to (successful) production. Kassel clarifies that the term 'intentional' has to be understood in the sense of prior intention, which agrees with Definitions 1-3 as well. It follows that Kassel's definition also excludes from the realm of artifacts, and thus from that of technical artifacts, natural entities, and waste products. Furthermore, Kassel clarifies that an intentional production is successful depending on the embodiment within the artifact of the properties individuated at the time the author performed the production. Interestingly, Kassel recognizes that production can lead to artifacts also when it is only partially successful but does not indicate how success should be judged. Thus, the view proposed in this work seems to be more restrictive that our Definition 1: it requires production in the sense of physical modification of the physical entity. It also does not agree with the

\footnotetext{
${ }^{8}$ This possibility allows to define an ontology of non-physical instruments - tests and questionnaires - used in Neuropsychology to assess the state of subjects, see Batrancourt et al. (2010).
} 
ontological status of OA because in that work the relationship between physical entities and artifacts is not one of constitution but of identity: the artifact and the physical object are indeed a single entity. This follows from allowing the artifact to have both the properties of the physical object and those of the a functional object (Kassel, 2010, p. 241).

Note that Kassel's notion does not go as far as requiring that the production process is given beforehand since, according to Kassel (2010, Section 2.1), only the conceptual representation of the artifact is explicitly mentioned as prior to the process and nothing is added in the accompanying axiomatization to enforce previous knowledge about the production process. This means that an artisan working without a specific production plan produces an artifact whenever the outcome of his/her work satisfies the sought properties. From this and the lack of specific check actions to establish the success of a production process, we conclude that the notion of artifact in Kassel's approach resembles Definition 2, that of EA, but is cast in different terminology: Kassel does not refer to selected behaviors and selected situations. Influenced by the DOLCE ontology framework and the philosophical literature, the author takes the notion of capacity, and subsequently function, as central for the characterization of artifacts and, as we have seen, defines a technical artifact to be an artifact with an ascribed capacity.

The notion of technical property (quality or capacity) was introduced in Definition 1 but, as we mentioned, is there used as an unspecified restriction to a subclass of properties. Kassel is more explicit in this case and proposes to consider as technical property (capacity) any property that enables "an agent to perform an action by using the artifact" (Kassel, 2010, p. 229) or, from the viewpoint of the role theory, as a property to play the role of instrument in certain types of actions. This latter reading and the fact that, in the DOLCE terminology, these relate to states and not to properties, makes this view coherent with the background and motivations for Definition 2, which lead us to conclude that (Kassel, 2010) is a qualified version of the engineering view on artifacts as we have characterized it in this paper.

\section{Conclusion}

We presented three perspectives on how to introduce the general notion of technical artifact in formal ontologies. We showed how the definitions of technical artifacts by these perspectives hang together and differ. From each perspective, technical artifacts form a category disjoint to natural entities but this disjointness is captured differently. It can be achieved by having technical artifacts be constituted by natural entities, or by having them made from natural entities by modification and production, bestowing on artifacts additional intentional properties, where again perspectives differ by the choice of this demarcating intentional property. Our presentation defines further work. The perspectives may be captured in more detail, and their relations can be given in more detail. A final issue is how to evaluate this plurality of perspectives. When all these perspectives are clarified enough to be accommodated in formal ontologies, the question is raised whether all should be accommodated, or only some of them.

\section{Acknowledgements}

This work has been developed in part within the Marie Curie EuJoint project (IRSES 247503). Stefano Borgo was also supported by the Gecko project, part of 'La Fabbrica del Futuro' (Programma Nazionale della Ricerca 2011-13). Pawel Garbacz was also supported by the grant N N101 150037 from the Ministry of Science and Higher Education. Yoshinobu Kitamura was also supported by Grants-in-Aid for 
Scientific Research (22300053) from the Ministry of Education, Culture, Sports, Science and Technology, Japan. Research by Pieter Vermaas was supported by the Netherlands Organization for Scientific Research (NWO).

\section{References}

Batrancourt, B., Dojat, M., Gibaud, B. \& Kassel, G. (2010). A core ontology of instruments used for neurological, behavioral and cognitive assessments. In Proceedings of the 6th International Conference on Formal Ontology in Information Systems, (FOIS'2010), Toronto (pp. 185-198). IOS Press.

Borgo, S., Franssen, M., Garbacz, P., Kitamura, Y., Mizoguchi, R. \& Vermaas, P.E. (2011). Technical Artifact: an Integrated Perspective. Proceeding of the Workshop Formal Ontologies Meet Industry (FOMI 2011), FAIA 229 (pp. 3-15). IOS Press.

Borgo, S. \& Masolo, C. (2009). Foundational choices in DolCE. In S. Staab and R. Studer (Eds.), Handbook on Ontologies. International Handbooks on Information Systems (pp. 361-381). Springer.

Borgo, S. \& Vieu, L. (2009). Artifacts in formal ontology. In A. Meijers (Ed.), Handbook of the Philosophy of Science. Handbook of Philosophy of Technology and Engineering Sciences (Vol. 9, pp. 273-307). Elsevier.

Burek, P. (2007). Ontology of functions: A domain-independent framework for modeling functions. PhD thesis. Available at: http://www.onto-med.de/publications/2007/burek-p-2007-a.pdf.

Burek, P., Herre, H. \& Loebe, F. (2009). Ontological analysis of functional decompositions. In Proceedings of the 8th International Conference on Software Methodologies, Tools and Techniques (SOMET-2009).

Carrara, M., Garbacz, P. \& Vermaas, P.E. (2011). If engineering function is a family resemblance concept: assessing three formalization strategies. Applied Ontology, 6(2), 141-163.

Carrara, M. \& Vermaas, P.E. (2009). The fine-grained metaphysics of artifactual and biological functional kinds. Synthese, 169, $125-143$.

Dipert, R.R. (1993). Artefacts, Art Works, and Agency. Temple University Press.

Hilpinen, R. (1992). Artefacts and works of art. Theoria, 58, 58-82.

Hilpinen, R. (1993). Authors and artefacts. Proceedings of the Aristotelian Society, 9, 155-178.

Houkes, W. \& Vermaas, P.E. (2009a). Contemporary engineering and the metaphysics of artefacts: beyond the artisan model. Monist, 92, 403-419.

Houkes, W. \& Vermaas, P.E. (2009b). Produced to use: combining two key intuitions on the nature of artefacts. Techne, 13, 123-136.

Houkes, W. \& Vermaas, P.E. (2010). Technical Functions: on the Use and Design of Artefacts. Springer.

Houkes, W. \& Vermaas, P.E. (2014). On what is made: instruments, products and natural kinds of artefacts. In M. Franssen, P. Kroes, T.A.C. Reydon and P.E. Vermaas (Eds.), Artefact Kinds: Ontology and the Human-Made World (pp. 167-190). Springer.

Kassel, G. (2010). A formal ontology of artefacts. Applied Ontology, 3,4, 223-246.

Kitamura, Y. \& Mizoguchi, R. (2010). Characterizing functions based on ontological models from an engineering point of view. In A. Galton and R. Mizoguchi (Eds.), Formal Ontology in Information Systems (pp. 301-314). IOS Press.

Kroes, P.A. \& Meijers, A.W.M. (2006). The dual nature of technical artifacts. Studies in History and Philosophy of Science, $37(1), 1-4$.

Masolo, C., Borgo, S., Gangemi, A., Guarino, N., Oltramari, A. \& Schneider, L. (2002). The wonderweb library of foundational ontologies. In Deliverable (Vol. 17). WonderWeb European Project, 2002.

Vieu, L., Borgo, S. \& Masolo, C. (2008). Artefacts and roles: modeling strategies in a multiplicative ontology. In C. Eschenbach and M. Gruninger (Eds.), Proceedings of the 5th FOIS Conference (pp. 121-134). IOS Press. 